Chemotherapy-induced cognitive deficits in patients with breast cancer, predominantly in attention and verbal memory, have been observed in numerous studies. These neuropsychological findings are corroborated by the results of neuroimaging studies. The aim of this paper was to survey the reports on cerebral structural and functional alterations in women with breast cancer treated with chemotherapy (CTx). First, we discuss the host-related and disease-related mechanisms underlying cognitive impairment after CTX. We point out the direct and indirect neurotoxic effect of cytostatics, which may cause: a damage to neurons or glial cells, changes in neurotransmitter levels, deregulation of the immune system and/or cytokine release. Second, we focus on the results of neuroimaging studies on brain structure and function that revealed decreased: density of grey matter, integrity of white matter and volume of multiple brain regions, as well as their lower activation during cognitive task performance. Finally, we concentrate on compensatory mechanisms, which activate additional brain areas or neural connection to reach the premorbid cognitive efficiency.

Key words: neuroimaging, cognition, breast cancer, chemotherapy.

Contemp Oncol (Pozn) 2017; 21 (1): 6-15 DOI: https://doi.org/10.5114/wo.2017.66652

\section{A systemic literature review of neuroimaging studies in women with breast cancer treated with adjuvant chemotherapy}

\author{
Paulina Andryszak $^{1}$, Monika Wiłkość ${ }^{1,2}$, Paweł Izdebski $^{1}$, Bogdan Żurawski ${ }^{3}$
}

${ }^{1}$ Institute of Psychology, Kazimierz Wielki University in Bydgoszcz, Poland

2Department of Psychiatry, Collegium Medicum in Bydgoszcz, Nicolaus Copernicus University in Torun, Bydgoszcz, Poland

${ }^{3}$ The Franciszek Lukaszczyk Oncology Center, Bydgoszcz, Poland

\section{Introduction}

The results of neuropsychological examinations carried out over the last two decades indicate the occurrence of cognitive impairments in patients with breast cancer who received chemotherapy [1]. A recent metaanalysis [2] showed a decrease in capacity of attention and selective attention as well as in immediate and delayed verbal recall in patients treated with chemotherapy compared to healthy persons. Changes observed during neuropsychological testing are corroborated by the results of neuroimaging studies carried out in the recent years [3-18].

The aim of this paper is to analyse the results of the neuroimaging studies conducted to date, assessing the cerebral alterations of women with breast cancer treated with chemotherapy.

The paper first focuses on the mechanisms underlying the cognitive decline, then describes the results of the studies on the structural and functional changes in the brain, and finally reports on the compensatory mechanisms observed in chemotherapy-treated women with breast cancer.

\section{Mechanisms of chemotherapy-induced cognitive impairments}

The mechanisms of cognitive impairment after chemotherapy (CTx) are still not fully understood $[19,20]$. The potential role of various factors is indicated, related both to individual characteristics (host-related, soil characteristics) and the neoplastic disease itself (disease-related, seed characteristics) [21].

Research results imply a direct neurotoxic effect of cytostatic agents, which cross the blood-brain barrier causing, for exzample damage to neurons or glial cells, changes in neurotransmitter levels [22-26], and microvascular damage related to ischemia and brain damage, such as decreased vascular density in the hippocampus after the use of methotrexate [19, 27]. The indirect mechanisms are associated with the deregulation of the immune system and/or release cytokines [22, 28, 29], hormonal changes, e.g., decreased levels of oestrogen and progesterone due to premature menopause [30,31], or DNA damage due to the effect of oxidative stress and accelerated telomere shortening $[22,28]$. Moreover, the significance of individual factors associated with age, vascular risk factors, or the pre-cancer level of cognitive functioning and the amount of cognitive reserves, is also pointed out [31].

The results of more recent studies indicate that some patients may exhibit genetic predisposition to cognitive impairments [20,31]. A relationship has been shown between the allele $\varepsilon 4$ of apolipoprotein E (APOE) gene and the deterioration of cognitive functioning in patients previously treated for 
breast cancer or lymphoma [26]. It was also found that persons with the catechol-O-methyltransferase (COMT)-Val genotype are more susceptible to the negative effects of CTx on cognitive functioning [32]. Genetic polymorphism may be related to the effectiveness of the blood-brain barrier (e.g. different expression of the multidrug resistance gene encoding P-glycoprotein, MDR1), the functioning of cytokines (e.g. polymorphism of the interleukin 6 cytokine gene), neurotransmitters (e.g. the polymorphism of COMT gene), and DNA repair mechanisms (e.g. the polymorphism of the $X$-ray repair cross complementing protein gene, XRCC1) [22, 33].

\section{Methods}

A comprehensive literature search was conducted using the PubMed database. The following search terms and their derivatives were used: cognition, neuroimaging, fMRI, PET, MRI, chemotherapy, breast cancer. Studies had to assess brain functioning with neuroimaging methods, be published in a peer-reviewed journal, and be available as full text in English language. No time period was specified.

\section{Results}

Forty-one studies fulfilled the inclusion criteria and were selected for further analysis. Changes in the central nervous system of women with breast cancer (BC) treated with CTx were assessed in 15 studies using an MRI [6, 10$13,34-43$ ] and in 24 studies using functional neuroimaging methods [3, 5, 7-9, 14-18, 44-57]. In two studies both structural and functional changes were assessed $[4,58]$. The characteristics of structural and functional studies in breast cancer patients are presented in Tables 1-4.

\section{Structural changes in the central nervous system of women with breast cancer treated with chemotherapy}

In ten studies researchers used Voxel-Based Morphometry (VBM) $[4,6,12,37-42,58]$ to compare the volume of brain areas and the density of grey and white matter [59]. In five studies Diffusion-Tensor Imaging (DTI) [11, 35, 36, 38, 43] was used to measure the microstructural integrity of white matter using fractional anisotropy (FA) and structural connectivity of the brain [60] was applied. In

Table 1. Structural cerebral changes in breast cancer patients prior and after chemotherapy

\begin{tabular}{|c|c|c|}
\hline Assessment & Grey matter changes & White matter changes \\
\hline Prior to CTx & $\begin{array}{l}\leftrightarrow \text { hippocampal volume in BC CTx+ and CTx- [34] } \\
\leftrightarrow \text { density and volume of GM between BC CTx+/CTx-/HC [37, 41, 58] } \\
\leftrightarrow \text { GM volume in DLPFC and superior parietal cortex between BC } \\
\text { CTx+/CTx-/HC [58] } \\
\downarrow \text { left cingulate GM density in BC CTx- compared to HC [12] }\end{array}$ & $\begin{array}{l}\downarrow \text { WM volume in frontal, parietal and limbic } \\
\text { regions depending on type of analyses and } \\
\text { covariates entered [41] } \\
\downarrow \text { WM integrity in BC compared to HC [58] }\end{array}$ \\
\hline $\begin{array}{l}1-12 \text { months } \\
\text { after CTx }\end{array}$ & $\begin{array}{l}\downarrow \text { prefrontal, parahippocampal, cingulate gyrus, precuneus volume [6] } \\
\downarrow \text { bilateral frontal, temporal (including hippocampus and adjacent } \\
\text { medial temporal structures) and cerebellar regions and right thalamus } \\
\text { GM density in BC CTx+ than HC [37] } \\
\downarrow \text { left frontal CH density in BC CTx+ compared to HC [12] } \\
\downarrow \text { frontal, temporal, parietal, and occipital volume [42] }\end{array}$ & $\begin{array}{l}\downarrow \text { FA in frontal, parietal and occipital tracts } \\
\text { after CTX+[11,36] } \\
\downarrow \text { prefrontal, parahippocampal, cingulate } \\
\text { gyrus, precuneus volume [6] }\end{array}$ \\
\hline $\begin{array}{l}1-2 \text { years after } \\
\text { CTx }\end{array}$ & $\begin{array}{l}\downarrow \uparrow \text { bilateral superior frontal, left middle frontal, right superior } \\
\text { temporal and cerebellar GM density [37] } \\
\downarrow \text { persisted in bilateral cerebellum, right thalamus and medial } \\
\text { temporal lobe, left middle gyrus and right precentral, medial frontal } \\
\text { and superior frontal gyri [37] } \\
\downarrow \text { in bilateral frontal and temporal regions [42] } \\
\downarrow \text { global hippocampal volume in 8\% of BCS CTx+ [10] } \\
\downarrow \text { posterior hippocampus in 11\% BCS CTX+ compared to HC [10] }\end{array}$ & $\begin{array}{l}\downarrow \text { FA in genu of corpus callosum in BCS CTX+ } \\
\text { than HC [35] }\end{array}$ \\
\hline $\begin{array}{l}\text { 2-10 years } \\
\text { after CTx }\end{array}$ & $\begin{array}{l}\leftrightarrow \text { prefrontal, parahippocampal, cingulate gyrus, precuneus volume } \\
\text { between BCS CTx+/CTx-/HC [6] } \\
\downarrow \text { posterior cortical regions and cerebellum volume in BCS HCTx+ } \\
\text { compared to CTx- [38] } \\
\downarrow \text { small-world characteristics of GM; altered interactions in } \\
\text { frontotemporal regions; fewer network hubs in BCS CTx+ compared } \\
\text { to HC [40] } \\
\downarrow \text { left hippocampal volumes in BCS CTx+ compared to HC [13] }\end{array}$ & $\begin{array}{l}\downarrow \text { white matter integrity BCS HCTx+ } \\
\text { compared to CTx- [38] }\end{array}$ \\
\hline $\begin{array}{l}>10 \text { years } \\
\text { after } C T x\end{array}$ & $\begin{array}{l}\downarrow \text { total brain and GM volume in BSC CTX+ compared to reference } \\
\text { group [39] }\end{array}$ & $\begin{array}{l}\leftrightarrow \text { prevalence of infractions or WM lesions } \\
\text { volume in BSC CTX+ than reference group [43] } \\
\uparrow \text { prevalence of total cerebral microbleeds } \\
(\mathrm{CMBS}) \text { and CMBs in deep/infratentorial } \\
\text { regions in BSC CTX+ than reference group [43] }\end{array}$ \\
\hline
\end{tabular}


one study semi-automatic segmentation procedure was used [34] and in three automatic seqmentation procedure were used [10, 13, 39]. Most of the studies were conducted in cross-sectional design: 10 in breast cancer survivors treated with CTx $[4,6,10,13,34,35,38-40,43]$ and 2 in breast cancer patients prior to CTx $[41,58]$; 5 studies were conducted with longitudinal design [11, 36, 37, 61, 62]. The results obtained from breast cancer patients treated with CTx were compared to breast cancer patient without CTx [34, 38, 55], healthy controls $[4,10,13,35,40,41,62]$, non-cancer reference subjects $[39,43]$, or breast cancer patients without CTx and healthy controls [6, 36, 37, 58, 61]. In four studies breast cancer patients were treated with the same schema of CTx $[38,39,43,58]$ and in the other studies different schemas were applied [4, 6, 10-13, 34-37, $40,42]$. A summary of the structural cerebral changes described in analyzed studies is presented in Table 1.
The evaluation of the anatomical properties of the brain using MRI yields information on the structural changes occurring over time and makes it possible to discern the differences between groups. As already mentioned in the discussion of some of the studies, supplementing the research using MRI with functional imaging techniques is a method to obtain fuller descriptions of chemotherapyrelated cognitive impairment $(\mathrm{CRCl})$ [63].

\section{Functional changes in the central nervous system of women with breast cancer treated with chemotherapy}

The functional studies were carried out using fMRI [4, 5, 7, 8, 14-16, 18, 48-55, 57, 58, 64], EEG [44, 45, 65], resting state fMRI [3, 17], PET [9] and Pulsed Arterial Spin Labelling MRI Perfusion [56]. During $\mathrm{FMRI}$ cognitive processes were assessed using verbal and visual working memo-

Table 2. Functional changes in breast cancer patients prior to and after chemotherapy

\begin{tabular}{|c|c|}
\hline Assessment & Functional changes \\
\hline Prior to CTx & $\begin{array}{l}\uparrow \text { bifrontal and biparietal regions in high load task in BC compared to HC [48] } \\
\uparrow \text { inferior frontal gyrus, insula, thalamus and midbrain during working memory in BC [49] } \\
\uparrow \text { bifrontal and } \downarrow \text { left parietal in BC CTx+/CTx- compared to HC [8] } \\
\downarrow \text { cerebellar in BC than NCN [50] } \\
\uparrow \text { prefrontal with increasing task difficulty on a planning task compared to HC, but not during a memory task [58] } \\
\neq \text { neural response }-\uparrow \text { spatial variance in executive network activity [54] } \\
\leftrightarrow \text { in the multitasking network [55] } \\
\leftrightarrow \text { perfusion in GM between BC CTx+/CTx- [56] }\end{array}$ \\
\hline $\begin{array}{l}\text { 1-12 months after } \\
\text { CTx }\end{array}$ & $\begin{array}{l}\downarrow \text { in bifrontal regions in BC CTx+/CTx- [8] } \\
\downarrow \text { in left inferior frontal when comparing to prior CTx+ [8] } \\
\uparrow \text { in left thalamic and posterior middle temporal gyrus compared with HC and } \uparrow \text { in right cerebellar and left } \\
\text { inferior precentral and posterior middle temporal gyrus compared with the CTx- [8] } \\
\downarrow \text { bilateral insula, left inferior orbitofrontal cortex and left middle temporal gyrus [15] } \\
\uparrow \text { brain activity magnitude in BC CTx+ with CIA [52] } \\
\downarrow \text { functional connectivity [53] } \\
\downarrow \text { in the multitasking network [55] } \\
\downarrow \text { in frontospatial executive network and cognitive complaints [18] } \\
\uparrow \text { perfusion in superior and posterior regions in BC CTx+ not related with } \downarrow \text { frontal GM density, however } \downarrow \text { frontal } \\
\text { GM density was associate with } \downarrow \text { perfusion in bilateral frontal and parietal lobes [56] } \\
\neq \text { resting state functional connectivity in BCS women with subjective cognitive complaints [17] }\end{array}$ \\
\hline $1-2$ years after $C T x$ & $\begin{array}{l}\uparrow \text { in bifrontal and biparietal regions during cognitive task but not significant differences in test performance [46] } \\
\downarrow \uparrow \text { in left frontal region as prior to CTx; } \downarrow \text { persisted in middle frontal gyrus [8] } \\
\uparrow \text { partial return to baseline in the dorsal attention network [53] }\end{array}$ \\
\hline $2-10$ years after $C T x$ & $\begin{array}{l}\downarrow \text { earlier P3 component in BCS CTx+ [44, 65] } \\
\downarrow \text { amplitude of P3 component in BCS HCTx+ [45, 65] } \\
\neq \text { cerebral blood flow in frontal cortex and cerebellum during memory task in BCS CTx+ [9] } \\
\downarrow \text { left middle dorsolateral prefrontal cortex and premotor cortex in BCS CTx+ compared to HC, } \downarrow \text { left caudal } \\
\text { prefrontal cortex and worse test performance in BCS CTx+ compared to CTx- and HC [7] } \\
\downarrow \text { prefrontal and parietal areas in BCS CTx+ compared to CTx-; } \uparrow \text { frontal activation - better test performance [5] } \\
\neq \text { global brain network organisation: } \uparrow \text { global clustering, } \neq \text { regional network characteristics in frontal, striatal and } \\
\text { temporal areas [3] } \\
\downarrow \text { left precuneus connectivity in AC CTx+ and } \downarrow \text { verbal performance [16] } \\
\neq \text { default mode network resting-state functional connectivity [51] } \\
\downarrow \text { prefrontal cortex during encoding task [14] } \\
\uparrow \text { in right superior temporal gyrus extending into bilateral fusiform, bilateral lingual gyri, left hippocampus, } \\
\text { bilateral basal ganglia, right precentral gyrus, right superior and inferior frontal gyri, right middle frontal gyrus, } \\
\text { bilateral inferior frontal gyrus, right cingulate gyrus, bilateral insula, bilateral parahippocampal gyrus, bilateral } \\
\text { cuneus, bilateral precuneus, bilateral superior parietal lobe, and cerebellum during recall task [14] }\end{array}$ \\
\hline$>10$ years after CTx & $\downarrow$ prefrontal and parietal areas [57] \\
\hline
\end{tabular}


Table 3. Characteristics of structural studies in breast cancer patients

\begin{tabular}{|c|c|c|c|c|c|}
\hline Study & Population & $\begin{array}{l}\text { Major CTx } \\
\text { treatment }\end{array}$ & Design & $\begin{array}{l}\text { Neuroimaging } \\
\text { method }\end{array}$ & Major findings \\
\hline $\begin{array}{l}\text { Yoshikawa } \\
\text { et al. } 2005 \\
{[34]}\end{array}$ & $\begin{array}{l}44 \mathrm{BCS} C T \mathrm{~T}+\mathrm{A}: \\
48.3 \pm 5.7 ; 31 \mathrm{BCS} \\
\mathrm{CTX}-\mathrm{A}: 48.2 \pm 5.7\end{array}$ & $\begin{array}{l}\text { CMF 32\%; AC } \\
\text { 30\%; 5FU 21\%; }\end{array}$ & $\begin{array}{l}\text { CS; TSCTx } 1262 \\
\pm 396 \text { days }\end{array}$ & MRI; ASP & No differences in hippocampal volume \\
\hline $\begin{array}{l}\text { Inagaki et al. } \\
2007 \text { [6] }\end{array}$ & $\begin{array}{l}\text { S1: } 51 \text { BCS CTX+A: } \\
47.3 \pm 5.2 ; 54 \text { CTX-A: } \\
46.3 \pm 6.1 ; 55 \text { HC A: } \\
46.2 \pm 6.7 \text { S2: } 73 \text { BCS } \\
\text { CTx+A: } 48.2 \pm 5.6 ; \\
59 \text { BSC CTX-A: } 48.4 \\
\pm 4.8 ; 37 \text { HC A: } 48.0 \\
\pm 6.4\end{array}$ & $\begin{array}{l}\text { S1: CMS 78\%; } \\
\text { AC: } 6 \% \text {; UFT: } \\
\text { 10\%; S2: CMF: } \\
\text { 51\%; AC: } 20 \% \text {; } \\
\text { UFT: } 10 \%\end{array}$ & $\begin{array}{l}\text { CS: } \mathrm{S} 1<1 \text { year; } \\
\text { TSCTX } 119 \\
\text { days } \pm 47 \mathrm{~S} 2 \pm \\
3 \text { years; } \mathrm{TSCT} \times \\
1189 \text { days } \pm 359\end{array}$ & MRI; VBM & $\begin{array}{l}1 \text { year after treatment smaller GM WM in } \\
\text { prefrontal, parahippocampal, cingulate gyrus } \\
\text { and precuneus; these regions correlated with } \\
\text { indices of attention/concentration and/or visual } \\
\text { memory; } 3 \text { years after no differences in GM and } \\
\text { WM between CTx+, CTx- and HC }\end{array}$ \\
\hline $\begin{array}{l}\text { Abraham } \\
\text { et al. } 2008 \\
{[35]}\end{array}$ & $\begin{array}{l}10 \text { BCS CT+A:49.8 } \\
\pm 8.09 \text { HC A: } 46.8 \\
\pm 6.8\end{array}$ & $\begin{array}{l}\text { AC: } 50 \% \text {; AC-T: } \\
50 \%\end{array}$ & $\begin{array}{l}\text { CS; TSCTX: } 22 \\
\pm 10 \text { months }\end{array}$ & MRI; DTI & $\begin{array}{l}\text { Lower FA in the genu of the corpus callosum in } \\
\text { BCS than HC. Positive correlation between FA in } \\
\text { the genu and processing speed }\end{array}$ \\
\hline $\begin{array}{l}\text { Deprez et al. } \\
2010 \text { [36] }\end{array}$ & $\begin{array}{l}34 \mathrm{BC} C T \mathrm{~A}+\mathrm{A}: 43.7 \\
\pm 6.1 ; 16 \mathrm{BC} C \mathrm{CT}-\mathrm{A}: \\
43.1 \pm 5.7 ; 19 \mathrm{HC} \text { : } \\
43.8 \pm 4.9\end{array}$ & UNK & $\begin{array}{l}\text { L; T1: before } \\
\text { CTX; T2: } 3-4 \\
\text { months after } \\
\text { CTx }\end{array}$ & MRI; DTI & $\begin{array}{l}\text { Decreased FA in frontal, parietal and occipital } \\
\text { WM tracts in } C T x+\text { in } T 2 \text { compared to T1. No } \\
\text { changes in } C T x \text { - and } H C\end{array}$ \\
\hline $\begin{array}{l}\text { McDonald } \\
\text { et al. } 2010 \\
{[37]}\end{array}$ & $\begin{array}{l}17 \mathrm{BC} C \mathrm{CT}+\mathrm{A}: 52.4 \\
\pm 8.5 ; 12 \mathrm{BC} C \mathrm{CT}- \\
\mathrm{A}: 52.7 \pm 7.2 ; 18 \mathrm{HC} \mathrm{A}: \\
50.6 \pm 6.5\end{array}$ & $\begin{array}{l}\text { AC-T: } 71 \% ; A C: \\
\text { 18; TAC } 11 \%\end{array}$ & $\begin{array}{l}\text { L; BC CT+: T1: } \\
\text { before CTx; T2: } \\
1 \text { month after } \\
\text { CTx; T3: } 1 \text { year } \\
\text { after CTx; BC } \\
\text { CTx-/HC yoked } \\
\text { intervals }\end{array}$ & MRI; VBM & $\begin{array}{l}\text { Pre-chemotherapy no between-group differences } \\
\text { in } \mathrm{GM} 1 \text { month after } \mathrm{CTx} \text { reduced bilateral frontal, } \\
\text { temporal, and cerebella } \mathrm{GM} \text { density in } \mathrm{BC} \text { relative } \\
\text { to } \mathrm{HC} 1 \text { year after } \mathrm{CTx} \text { changes improved in some } \\
\text { regions and persisted with others } \mathrm{CT} \text { - reduced } \\
\text { right cerebellar } \mathrm{GM} \text { density relative to } \mathrm{HC} \text { in } \mathrm{T} 2\end{array}$ \\
\hline $\begin{array}{l}\text { Bergouignan } \\
\text { et al. } 2011 \\
{[10]}\end{array}$ & $\begin{array}{l}16 \text { BCS CTx+ A: } 48.7 \\
\pm 5.0 ; 21 \text { HC A: } 47.7 \\
\pm 5.3\end{array}$ & UNK & $\begin{array}{l}\text { CS; TSCT > } 18 \\
\text { months }\end{array}$ & MRI; ASP & $\begin{array}{l}\text { The global hippocampal volume reduces in } \\
8 \% \text { and posteriori hippocampus in } 11 \% \text { in } \mathrm{BC} \\
\text { compared to HC. Reduced autobiographical } \\
\text { memory related to posteriori hippocampal } \\
\text { volume }\end{array}$ \\
\hline $\begin{array}{l}\text { Deprez et al. } \\
2012 \text { [11] }\end{array}$ & $\begin{array}{l}34 \mathrm{BC} C \mathrm{CT}+\mathrm{A}: 43.7 \\
\pm 6.1 ; 16 \mathrm{BC} C \mathrm{CT}-\mathrm{A}: \\
43.1 \pm 5.7 ; 18 \mathrm{HC} \\
\mathrm{A}: 43.8 \pm 4.9\end{array}$ & $\begin{array}{l}\text { FEC: } 35 \% \text {; } \\
\text { FEC-T: } 65 \%\end{array}$ & $\begin{array}{l}\text { L; T1: before } \\
\text { CTX; T2: } 3-4 \\
\text { months after } \\
\text { CTx; CTX-/HC } \\
\text { yoked intervals }\end{array}$ & MRI; DTI & $\begin{array}{l}\text { Decrease of FS in frontal, parietal, and occipital } \\
\text { WM tracts after CTx compared baseline. Mean } \\
\text { regional FA changes correlated with attention } \\
\text { and verbal memory in BC CTx+ group }\end{array}$ \\
\hline $\begin{array}{l}\text { de Ruiter } \\
\text { et al. } 2012 \\
\text { [38] }\end{array}$ & $\begin{array}{l}17 \text { BCS CTx+A: } 56.5 \\
\pm 5.1 ; 15 \text { BCS CTx-A: } \\
58.2 \pm 5.8\end{array}$ & $\begin{array}{l}\mathrm{FEC}+\mathrm{CTC}+ \\
\text { autologous } \\
\text { peripheral blood } \\
\text { hematopoietic } \\
\text { progenitor-cell } \\
\text { transplantation } \\
\text { rescue } 100 \%\end{array}$ & $\begin{array}{l}\text { CS; TSCTX } 9.5 \\
\pm 0.8 \text { years }\end{array}$ & MRI; DTI; VBM & $\begin{array}{l}\text { Reduced GM volume in posteriori cortical regions } \\
\text { and cerebellum in CTX+ BCS compared to CTX-. } \\
\text { GM reduction in left posterior parietal cortex } \\
\text { overlapped with fMRI hypoactivation during } \\
\text { memory encoding and colocalised with WM } \\
\text { abnormalities. Reduced WM integrity in CTx+ }\end{array}$ \\
\hline $\begin{array}{l}\text { Hosseini } \\
\text { et al. } 2012 \\
{[40]}\end{array}$ & $\begin{array}{l}37 \text { BCS CTX+ A: } 54.2 \\
\pm 6.1 ; 38 \text { HC A: } 55.5 \\
\pm 9.0\end{array}$ & $\begin{array}{l}\text { AC-T: 43\%; AC: } \\
24 \% ; C T: 16 \%\end{array}$ & $\begin{array}{l}\text { CS; TSCTx: } 4.5 \\
\pm 3.4 \text { years }\end{array}$ & $\begin{array}{l}\text { MRI; VBM; Graph } \\
\text { theoretical } \\
\text { analysis of } \\
\text { GM structural } \\
\text { networks }\end{array}$ & $\begin{array}{l}\text { Reduced small-world characteristics of GM, } \\
\text { altered interactions in frontotemporal regions } \\
\text { and fewer networks hubs in } \mathrm{BC} \text { compared to } \mathrm{HC}\end{array}$ \\
\hline $\begin{array}{l}\text { Koppelmans } \\
\text { et al. } 2012 \\
{[39]}\end{array}$ & $\begin{array}{l}184 \mathrm{BCS} C \mathrm{CT}+\mathrm{A}: \\
64.0 \pm 6.5 ; 368 \text { non- } \\
\text { cancer reference } \\
\text { subjects } \mathrm{A}: 64.0 \pm 6.5\end{array}$ & CMF $100 \%$ & $\begin{array}{l}\text { CS; TSCTx: } 21.1 \\
\pm 4.4 \text { years }\end{array}$ & MRI; VBM; ASP & $\begin{array}{l}\text { Smaller total brain and GM volume in } \mathrm{BC} \\
\text { compared to reference subjects. Observed } \\
\text { smaller GM volume comparable to the effect of } \\
\text { almost } 4 \text { years of aging }\end{array}$ \\
\hline $\begin{array}{l}\text { Scherling } \\
\text { et al. } 2012 \\
{[41]}\end{array}$ & $\begin{array}{l}23 \text { BC CT }+ \text { A: } 51.0 \\
\pm 8.5 ; 23 \text { HC A: } 50.0 \\
\pm 9.0\end{array}$ & NA & $\begin{array}{l}\text { CS; BC prior to } \\
\mathrm{CTx}\end{array}$ & MRI; VBM & $\begin{array}{l}\text { No differences in GM between BC and HC. Lower } \\
\text { WM volumes in frontal, parietal and limbic } \\
\text { regions in BC than in HC. Findings modified by } \\
\text { inclusion of covariates }\end{array}$ \\
\hline $\begin{array}{l}\text { Conroy et al. } \\
2013 \text { [4] }\end{array}$ & $\begin{array}{l}24 \text { BCS CTx+ A: } 57.8 \\
\pm 9.6 ; 23 \text { HC A: } 61.2 \\
\pm 9.9\end{array}$ & $\begin{array}{l}\text { AC: } 29 \% \text {; AC-T: } \\
\text { 21\%; A-T: } 12 \%\end{array}$ & $\begin{array}{l}\text { CS; TSCTx } 6.4 \\
\pm 2.1 \text { years }\end{array}$ & MRI; VBM & $\begin{array}{l}\text { Decreased GM density in several brain regions } \\
\text { in BC compared to HC. GM density negatively } \\
\text { related to oxidative DNA damage and learning } \\
\text { and memory performance. Post CTx interval } \\
\text { positively related to right frontal GM density } \\
\text { (related to cognition) }\end{array}$ \\
\hline
\end{tabular}


Table 3. Cont.

\begin{tabular}{|c|c|c|c|c|c|}
\hline Study & Population & $\begin{array}{l}\text { Major CTx } \\
\text { treatment }\end{array}$ & Design & $\begin{array}{l}\text { Neuroimaging } \\
\text { method }\end{array}$ & Major findings \\
\hline $\begin{array}{l}\text { Kesler et al. } \\
2013 \text { [13] }\end{array}$ & $\begin{array}{l}42 \text { BC CTx+ A: } 54.6 \\
\pm 6.5 ; 35 \text { HC A: } 55.5 \\
\pm 9.3\end{array}$ & $\begin{array}{l}\text { AC or } \mathrm{CT}: 86 \% \\
5 \mathrm{FU}-\mathrm{T} \text { or } \mathrm{M}: \\
14 \%\end{array}$ & $\begin{array}{l}\mathrm{CS} ; \mathrm{TSCT} \times 4.8 \\
\pm 3.4 \text { years }\end{array}$ & $\begin{array}{l}\text { MRI; automated } \\
\text { hippocampal } \\
\text { segmentation }\end{array}$ & $\begin{array}{l}\text { Reduced left hippocampal volumes and elevated } \\
\text { interleukin- } 6 \text { and tumour necrosis factor } \alpha \text { in } \\
\text { BC compared to HC. Cytokine levels and left } \\
\text { hippocampal volume in both groups associated } \\
\text { with verbal memory performance }\end{array}$ \\
\hline $\begin{array}{l}\text { McDonald } \\
\text { et al. } 2013 \\
{[12]}\end{array}$ & $\begin{array}{l}27 \text { BCS CTx+ A: } 49.9 \\
\pm 7.6 ; 28 \text { BCS CTx-A: } \\
52.4 \pm 9.1 ; 24 \text { HC A: } \\
47.0 \pm 9.2\end{array}$ & $\begin{array}{l}\text { AC-T 33\%; } \\
\text { CT 33\%; D/ } \\
\text { carboplatin } \\
18 \%\end{array}$ & $\begin{array}{l}\text { L; T1: before } \\
\text { CTx; T2: } \\
1 \text { month after } \\
\text { CTx; BC CTx-/ } \\
\text { HC yoked } \\
\text { intervals }\end{array}$ & MRI; VBM & $\begin{array}{l}\text { Pre-chemotherapy reduced left cingulate GM } \\
\text { density in BCS CTx-compared to HC. } 1 \text { month } \\
\text { after } \mathrm{CTx} \text { reduced left frontal GM density in BCS } \\
\mathrm{CTx}+\text { compared to HC. Left frontal GM density } \\
\text { related to self-reported executive function }\end{array}$ \\
\hline $\begin{array}{l}\text { Lepage et al. } \\
2014 \text { [42] }\end{array}$ & $\begin{array}{l}19 \text { BC CTx+ A } 50.2 \\
\pm 8.6 ; 19 \text { HC A: } 49.3 \\
\pm 9.0\end{array}$ & FEC-D: 68\%; & $\begin{array}{l}\text { L; T1: before } \\
\text { CTx; T2: } \\
1 \text { month after } \\
\text { CTx; T3: } 1 \text { year } \\
\text { after CTx }\end{array}$ & MRI; VBM & $\begin{array}{l}\text { In BC group distributed GM volume reductions } \\
1 \text { month after } \mathrm{CTx} \text {, a partial recovery } 1 \text { year after } \\
\mathrm{CTx} \text { with persisted alterations in frontal and } \\
\text { temporal regions }\end{array}$ \\
\hline $\begin{array}{l}\text { Koppelmans } \\
\text { et al. } 2015 \\
{[43]}\end{array}$ & $\begin{array}{l}187 \mathrm{BCS} C T \mathrm{X}+\mathrm{A}: 64.1 \\
\pm 6.5 ; 374 \text { non-cancer } \\
\text { reference subject A: } \\
64.1 \pm 6.5\end{array}$ & CMF: $100 \%$ & $\begin{array}{l}\text { CS; TSCTx } 21.1 \\
4.3 \text { years }\end{array}$ & MRI; DTI & $\begin{array}{l}\text { Higher prevalence of total cerebral microbleeds } \\
\text { and in deep/infratentorial region in BSC than in } \\
\text { reference group. No differences in the prevalence } \\
\text { of infractions or WM lesion volume }\end{array}$ \\
\hline $\begin{array}{l}\text { Menning } \\
\text { et al. } 2015 \\
{[58]}\end{array}$ & $\begin{array}{l}32 \mathrm{BC} C T \mathrm{~A}+\mathrm{A}: 50.2 \\
\pm 9.2 ; 33 \mathrm{BC} C \mathrm{CT}-\mathrm{A}: \\
52.4 \pm 7.3 ; 38 \mathrm{HC} \text { : } \\
50.1 \pm 8.7\end{array}$ & anthracycline & CS; before CTx & MRI; VBM & $\begin{array}{l}\text { Lower WM integrity in BC compared to HC. } \\
\text { Alterations associated with symptoms of fatigue. } \\
\text { No differences in regional GM and WM volumes. } \\
\text { No differences in GM volume of ROIs in the } \\
\text { DLPFC and superior parietal cortex between } \\
\text { groups }\end{array}$ \\
\hline
\end{tabular}

CTX+- cancer patients treated with standard dose of chemotherapy; HCTX+-cancer patients treated with high-dose chemotherapy; CTX--cancer patients without chemotherapy; $B C$ - breast cancer patients; $B C S$ - breast cancer survivors; $H C$ - healthy controls; SRCI - self-reported cognitive impairment; $A$ - mean \pm SD age; $T S C T X$ - mean $\pm S D$ age time since chemotherapy; DTI - diffusion tensor imaging; MRI - magnetic resonance imaging; FA - fractional anisotropy; VBM voxel based morphometry; ASP - automatic segmentation procedure; ROI - region of interes; GR - gray matter; WM - white matter; CS - cross-sectional; L - longitudinal; $T$ - time point; $S$-sample

Adjuvant chemotherapy: CMF-cyclophosphamide, methotrexate, fluorouracil; AC-cyclophosphamide, doxorubicin; CAF-fluorouracil, cyclophosphamide, doxorubicin; 5FU - fluorouracil; UFT - tegafur, uracil; AC-T - AC followed by a taxane; TAC - docetaxel, doxorubicin, cyclophosphamide; A-T - doxorubicin, taxane; $C T$-cyclophosphamide, paclitaxel; CD - cyclophosphamide, docetaxel; D-docetaxel; M methotrexate; UNK - not known; NA - not applicable

ry tasks $[4,8,18,46,48,49,52-54]$, visual memory task $[5,57]$, verbal memory task $[9,14-16]$, attention [53] and executive functioning $[5,7,41,55,57]$. Most studies were conducted in cross-sectional design: 14 in breast cancer survivors treated with CTx $[3-5,7,9,14,16,17,44-46,51$, $57,65]$ and 4 in breast cancer patient prior to CTx [48-50, 58]; 8 studies were conducted in longitudinal design [8, $15,18,52-56]$. The results obtained by breast cancer patients treated with CTx were compared with breast cancer patients without CTx [7, 8, 16, 18, 38, 44, 45, 54-56], with breast cancer patients treated with different schemas of CTx $[9,16,45,57,65]$, with patients treated with radiotherapy [57], with healthy controls [3, 4, 8, 14, 15, 18, 46, 48, $52,57]$, or non-cancer reference subjects $[49,50]$ or with breast cancer patients without CTx and healthy controls [7-9, 18, 51, 54-56]. In three studies breast cancer patients were treated with the same schema of CTx [44, 45, 65], and in the others studies different schemas were applied [3, 4, 7, 8, 14-18, 45-48, 50-57].

Summary of functional changes described in the analysed studies (Table 2).

\section{Compensatory mechanisms}

An interesting study to observe the mechanism underlying the process of coping with cognitive demand was per- formed on 60-year-old homozygous twin sisters [46]. One of the sisters was previously (22 months earlier) treated for breast cancer that $\mathrm{AC}+\mathrm{T}$ adjuvant chemotherapy (four cycles of AC followed by four cycles of $T$ - docetaxel), and received hormonal therapy (tamoxifen) during the study. While diseases and therapies which could negatively affect cognitive functioning were excluded in both sisters, they were found to have the allele $\varepsilon 4$ of apolipoprotein $E$, associated with the occurrence of cognitive deficits [26]. Cognitive functioning was evaluated using standard neuropsychological tests, a self-assessment questionnaire, and functional magnetic resonance imaging (fMRI). It was found that the twin treated with CTx reported much greater problems with cognitive functioning. Nevertheless, the results of the performed neuropsychological tests lay within the norm and differed minimally from those of the healthy sister. The fMRI results showed white matter hyperintensities in both sisters, which are also observed among the carriers of the allele $\varepsilon 4$ of apolipoprotein $E$ [ 66 , 67]. No coherent pattern of the differences in the volumes of selected brain areas (including the hippocampus, amygdala, frontal part of the hippocampal gyrus cortex, and corpus callosum) were found between sisters. Nonetheless, interesting results were obtained in an $\mathrm{fMRI}$ examination during the performance of a task evaluating working 
Table 4. Characteristics of functional neuroimaging studies in breast cancer patients

\begin{tabular}{|c|c|c|c|c|c|}
\hline Study & Population & $\begin{array}{l}\text { Major CTx } \\
\text { treatment }\end{array}$ & Design & NI/NP methods & Major findings \\
\hline $\begin{array}{l}\text { Kreukels } \\
\text { et al. } 2005 \\
{[44]}\end{array}$ & $\begin{array}{l}26 \text { BCS CT+ A: } 51.5 \\
\pm 5.6 ; 23 \text { BCS CT-A: } \\
53.2 \pm 8.5\end{array}$ & CMF: $100 \%$ & $\begin{array}{l}\text { CS; } T S C T x: \text { CTx+: } \\
5.1 \text { years; } C T x-: \\
3.6 \text { years }\end{array}$ & EEG & $\begin{array}{l}\text { Differences in latency and amplitude of P3 } \\
\text { component between BCS CTX+ and CTx-. } \\
\text { Earlier and reduced P3 in CTX+ }\end{array}$ \\
\hline $\begin{array}{l}\text { Kreukels } \\
\text { et al. } 2006 \\
{[45]}\end{array}$ & $\begin{array}{l}12 \text { BCS HCTx+A: } \\
51.5 \pm 5.6 ; 17 \text { BCS } \\
\text { CTx+A: } 51.2 \pm 5.9 \\
23 \text { BCS CTx-A: } \\
53.2 \pm 8.5\end{array}$ & $\begin{array}{l}\text { HCTX+: FEC-CTC } \\
\text { with autologous } \\
\text { peripheral blood } \\
\text { hematopoietic } \\
\text { progenitor cell } \\
\text { transplantation; } \\
\text { CTx+: FEC } 100 \%\end{array}$ & $\begin{array}{l}\text { CS; TSCTx: HCTX+: } \\
3.7 \pm 0.8 \text { years; } \\
\text { CTx+: } 4.1 \pm 0.7 \\
\text { years }\end{array}$ & EEG & $\begin{array}{l}\text { Reduced amplitude of the P3 component } \\
\text { in BCS treated with with high dose } \\
\text { chemotherapy compared with BCS without } \\
\text { CTX }\end{array}$ \\
\hline $\begin{array}{l}\text { Ferguson } \\
\text { et al. } 2007 \\
{[46]}\end{array}$ & $\begin{array}{l}2 \text { monozygotic } \\
\text { twins } \mathrm{A}: 60 \text { years; } \\
\text { Twin } \mathrm{A} \text { : } \mathrm{BC} \text { CTX+ } \\
\text { Twin } \mathrm{B}: \mathrm{HC}\end{array}$ & $A C-D$ & $\begin{array}{l}\text { CS; TSCTx: } 22 \\
\text { months }\end{array}$ & $\begin{array}{l}\text { fMRI; verbal } \\
\text { N-back task }\end{array}$ & $\begin{array}{l}\text { Broader spatial extent of activation } \\
\text { in typical memory circuitry (bifrontal } \\
\text { and biparietal regions), more cognitive } \\
\text { complaints in BC twin. Small differences in } \\
\text { neuropsychological test performance }\end{array}$ \\
\hline $\begin{array}{l}\text { Silverman } \\
\text { et al. } 2007 \\
\text { [9] }\end{array}$ & $\begin{array}{l}5 \text { BCS CTX }+ \text { A: } 47.6 \\
\pm 6.0 ; 11 \text { BCS CTX+ } \\
\text { TAM A: } 51.7 \pm 4.7 \\
5 \text { BCS CTX-A: } 53.2 \\
\pm 4.1 ; 3 \text { HC A: } 57.9 \\
\pm 7.1\end{array}$ & UNK & $\begin{array}{l}\text { CS; TSCTx: 5-10 } \\
\text { years }\end{array}$ & $\begin{array}{l}\text { PET; verbal } \\
\text { memory task }\end{array}$ & $\begin{array}{l}\text { Altered cerebral blood flow in frontal } \\
\text { cortex and cerebellum during memory task } \\
\text { in BCS CTx+. Altered cerebral activation in } \\
\text { inferior frontal gyrus in CTx+. Correlation } \\
\text { between resting metabolism and task } \\
\text { performance }\end{array}$ \\
\hline $\begin{array}{l}\text { Kreukels } \\
\text { et al. } 2008 \\
{[65]}\end{array}$ & $\begin{array}{l}17 \text { BCS FEC CTX+ A: } \\
51.2 \pm 5.9 ; 12 \text { BSC } \\
\text { CTC CTx+ A: } 51.5 \\
\pm 5.6 ; 24 \text { BCS CMF } \\
\text { CTx+ A: } 51.4 \pm 5.7 ; \\
23 \text { BCs CTx- A: } 53.2 \\
\pm 8.5\end{array}$ & $\begin{array}{l}\text { FEC: } 100 \% \text {; FEC/ } \\
\text { CTC: } 100 \% \text { CMF: } \\
100 \%\end{array}$ & CS; TSCTx: 3-6 y & EEG & $\begin{array}{l}\text { Lower P3 amplitudes in BCS CTX+ than } \\
\text { in BCS CTX-. Differences in P3 latency } \\
\text { between BCS treated with different CTX+ } \\
\text { regimes }\end{array}$ \\
\hline $\begin{array}{l}\text { Kesler et al. } \\
2009 \text { [14] }\end{array}$ & $\begin{array}{l}14 \text { BCS CTX }+ \text { A: } 55.1 \\
\pm 8.0 ; 18 \text { HC A: } 54.2 \\
\pm 8.0\end{array}$ & $\begin{array}{l}\text { CMF: } 36 \% \text { AC-T: } \\
64 \%\end{array}$ & $\begin{array}{l}\text { CS; TSCTx: } 3.3 \\
\pm 3.3 \text { years }\end{array}$ & $\begin{array}{l}\text { fMRI; verbal } \\
\text { declarative } \\
\text { memory task }\end{array}$ & $\begin{array}{l}\text { Reduced activation in prefrontal cortex } \\
\text { during encoding task and increased } \\
\text { activation in multiple diffuse brain regions } \\
\text { during recall task in } \mathrm{BC} \text { compared to } \mathrm{HC}\end{array}$ \\
\hline $\begin{array}{l}\text { Cimprich } \\
\text { et al. } 2010 \\
{[48]}\end{array}$ & $\begin{array}{l}10 \text { BC CTx+ A: } 45 \\
\pm 89 \text { HC A: } 52 \pm 10\end{array}$ & NA & CS; before CTx & $\begin{array}{l}\text { fMRI; verbal } \\
\text { working memory } \\
\text { test }\end{array}$ & $\begin{array}{l}\text { Increased bifrontal and biparietal } \\
\text { activation at high task load in BC before } \\
\text { CTx compared to HC }\end{array}$ \\
\hline $\begin{array}{l}\text { Kesler et al. } \\
2011 \text { [7] }\end{array}$ & $\begin{array}{l}25 \mathrm{BC} C T \mathrm{x}+\mathrm{A}: 56.2 \\
\pm 7.8 ; 19 \mathrm{BC} C \mathrm{CT}-\mathrm{A}: \\
58.1 \pm 6.5 ; 18 \mathrm{HC} \mathrm{A}: \\
55.6 \pm 9.4\end{array}$ & $\begin{array}{l}\text { CTA or CA: } 36 \% \text {; } \\
\text { AC: } 28 \% ; \text { CMF: } \\
12 \%\end{array}$ & $\begin{array}{l}\text { CS; TSCTx: } 4.7 \\
\pm 5.9 \text { years }\end{array}$ & $\begin{array}{l}\text { fMRI; Wisconsin } \\
\text { card sorting }\end{array}$ & $\begin{array}{l}\text { Reduced activation in the left middle } \\
\text { dorsolateral prefrontal cortex and } \\
\text { premotor cortex in } \mathrm{BC} \text { compared to } \mathrm{HC} \text {. } \\
\text { Reduced left caudal lateral prefrontal } \\
\text { cortex activation and increased } \\
\text { perseverative errors and reduced } \\
\text { processing speed in } \mathrm{BC} C \mathrm{C} x+\text { compared to } \\
\mathrm{BC} \mathrm{CT} \text { - and } \mathrm{HC}\end{array}$ \\
\hline $\begin{array}{l}\text { de Ruiter } \\
\text { et al. } 2011 \\
\text { [5] }\end{array}$ & $\begin{array}{l}19 \text { BCS CTX+ A } 56.3 \\
\pm 5.5 ; 15 \text { BCS CTX- } \\
58.2 \pm 5.8\end{array}$ & $\begin{array}{l}\text { FEC-CTC with } \\
\text { autologous } \\
\text { peripheral blood } \\
\text { hematopoietic } \\
\text { progenitor cell } \\
\text { transplantation: } \\
100 \%\end{array}$ & $\begin{array}{l}\text { CS; TSXTx: } 9.8 \\
\pm 0.8 \text { years }\end{array}$ & $\begin{array}{l}\text { fMRI; Tower of } \\
\text { London; Paired } \\
\text { associates task }\end{array}$ & $\begin{array}{l}\text { Reduced prefrontal and parietal activation } \\
\text { in BC CTX }+ \text { compared to BCS CTX-. In BC } \\
C T x+\text { greater frontal activation related to } \\
\text { better performance in Tower of London } \\
\text { task }\end{array}$ \\
\hline $\begin{array}{l}\text { Scherling } \\
\text { et al. } 2011 \\
{[49]}\end{array}$ & $\begin{array}{l}23 \text { BC A: } 51.5 \pm 8.47 \\
23 \text { NCN A: } 50.4 \\
\pm 8.82\end{array}$ & NA & CS; before CTx & $\begin{array}{l}\text { fMRI; visuospatial } \\
\text { n-back task }\end{array}$ & $\begin{array}{l}\text { Increased activity in inferior frontal gyrus, } \\
\text { insula, thalamus and midbrain during } \\
\text { working memory in BC compared to } \\
\text { NCN. Findings modified by inclusion of } \\
\text { covariates }\end{array}$ \\
\hline $\begin{array}{l}\text { Bruno et al. } \\
2012 \text { [3] }\end{array}$ & $\begin{array}{l}34 \text { BCS CTx+ A: } \\
55.16 \pm 7.3 ; 27 \text { HC A: } \\
55.08 \pm 9.12\end{array}$ & $\begin{array}{l}\text { ACT: 79\%; CMF: } \\
\text { 15; AC + CMF: } 9 \%\end{array}$ & $\begin{array}{l}\text { CS; TSXTx: } 5.35 \\
\pm 5.40 \text { years }\end{array}$ & Resting state fMRI & $\begin{array}{l}\text { Alteration in functional brain networks } \\
\text { supporting executive functioning, memory } \\
\text { and emotion regulation in BC CTx+ } \\
\text { compared to HC. No correlation between } \\
\text { functional brain network, objective and } \\
\text { subjective cognitive measures }\end{array}$ \\
\hline
\end{tabular}


Table 4. Cont.

\begin{tabular}{|c|c|c|c|c|c|}
\hline Study & Population & $\begin{array}{l}\text { Major CTx } \\
\text { treatment }\end{array}$ & Design & NI/NP methods & Major findings \\
\hline $\begin{array}{l}\text { McDonald } \\
\text { et al. } 2012 \\
{[8]}\end{array}$ & $\begin{array}{l}16 \text { BC CTX }+ \text { A: } 52.9 \\
\pm 8.6 ; 12 \text { CTX-A: } \\
52.7 \pm 7.2 ; 15 \text { HC A: } \\
50.5 \pm 6.0\end{array}$ & $\begin{array}{l}\text { ACT: 69\%; TAC: } \\
12 \% ; A C: 19 \%\end{array}$ & $\begin{array}{l}\text { L; T1: before CTx; } \\
\text { T2: } 1 \text { month after } \\
\text { CTx; T3: } 1 \text { year } \\
\text { after CTx }\end{array}$ & $\begin{array}{l}\text { fMRI; verbal } \\
\text { N-back task }\end{array}$ & $\begin{array}{l}\text { In both } \mathrm{BC} \text { groups increased activation in } \\
\text { bifrontal in } \mathrm{T} 1 \text {, reduced activation at } \mathrm{T} 2 \text {; } \\
\text { in some regions return to baseline at } \mathrm{T3} \\
\text { - suggesting compensatory recruitment } \\
\text { during working memory task. In } \mathrm{BC} C \mathrm{C} x+ \\
\text { decreased left frontal activation in T2 } \\
\text { comparing to T1 but returning to baseline } \\
\text { at T3 - possible effect of CTx }\end{array}$ \\
\hline $\begin{array}{l}\text { Scherling } \\
\text { et al. } 2012 \\
{[50]}\end{array}$ & $\begin{array}{l}23 \text { BC A: } 51.5 \pm 8.47 \\
23 \text { NCN A: } 50.4 \\
\pm 8.82\end{array}$ & NA & CS; before CTx & $\begin{array}{l}\text { fMRI; go/no-go } \\
\text { task }\end{array}$ & $\begin{array}{l}\text { In BC less activity in cerebellar area than } \\
\text { in NCN }\end{array}$ \\
\hline $\begin{array}{l}\text { Kesler et al. } \\
2013[51]\end{array}$ & $\begin{array}{l}30 \text { BCS CTX+A: } 55 \\
\pm 7 ; 27 \text { BCS CTX- A: } \\
58 \pm 7 ; 24 \text { HC A: } \\
56 \pm 9\end{array}$ & AC-T: $87 \%$ & $\begin{array}{l}\mathrm{CS} ; \mathrm{TSCT} \times 4.5 \pm 3.3 \\
\text { years }\end{array}$ & $f M R I$ & $\begin{array}{l}\text { Default mode network resting state } \\
\text { functional connectivity patterns disturbed } \\
\text { in } \mathrm{BCS} C T \mathrm{x}+\mathrm{t}\end{array}$ \\
\hline $\begin{array}{l}\text { López Zunini } \\
\text { et al. } 2013 \\
\text { [15] }\end{array}$ & $\begin{array}{l}21 \text { BC A: } 50.62 \\
\pm 8.37 ; 21 \text { HC A: } \\
49.67 \pm 8.75\end{array}$ & $\begin{array}{l}\text { FECT: } 62 \% \text {; CD: } \\
\text { 19\% AC: } 19 \%\end{array}$ & $\begin{array}{l}\text { L; T1: before CTx; } \\
\text { T2: } 1 \text { m after CTx }\end{array}$ & $\begin{array}{l}\mathrm{fMRI} \text {; verbal } \\
\text { memory recall }\end{array}$ & $\begin{array}{l}\text { In } \mathrm{BC} \text { decrease activation in the bilateral } \\
\text { insula, the left inferior orbitofrontal } \\
\text { cortex and the left middle temporal gyrus } \\
\text { post-chemotherapy in compared to pre- } \\
\text { chemotherapy, and to HC }\end{array}$ \\
\hline $\begin{array}{l}\text { Conroy et al. } \\
2013[4]\end{array}$ & $\begin{array}{l}24 \mathrm{BSC} C \mathrm{CT}+\mathrm{A}: \\
57.8 \pm 9.6 ; 23 \mathrm{HC} \text { A: } \\
61.2 \pm 9.9\end{array}$ & AC: $29 \%$ & $\begin{array}{l}\text { AC-T: } 21 \% ; A-T: \\
12 \%\end{array}$ & $\begin{array}{l}\text { CS; TSCTx: } 6.42 .1 \\
\text { years }\end{array}$ & $\begin{array}{l}\mathrm{fMRI} \text {; visual } n \text {-back } \\
\text { Lower activation in several regions in BCS. } \\
\text { Activation in the right anterior frontal } \\
\text { region positively correlated with post- } \\
\text { chemotherapy interval }\end{array}$ \\
\hline $\begin{array}{l}\text { Conroy et al. } \\
2013[52]\end{array}$ & $\begin{array}{l}9 \text { BC CTx+ with CIA } \\
\text { A: } 45.3 \pm 5.8 \\
6 \text { BC CTx+ post- } \\
\text { menopausal A: } \\
58.7 \pm 4.4 ; 6 \mathrm{HC} \\
\text { pre-menopausal } \\
\text { A: } 44.8 \pm 4.0 ; 6 \mathrm{HC} \\
\text { post-menopausal } \\
\text { A: } 55.2 \pm 4.0\end{array}$ & $\begin{array}{l}\text { AC-T: } 78 \% ; A C: \\
11 \%\end{array}$ & $\begin{array}{l}\text { L; T1: before CTx; } \\
\text { T2: } 1 \text { month after }\end{array}$ & $\begin{array}{l}\text { fMRI; visual } \\
\text { n-back }\end{array}$ & $\begin{array}{l}\text { Increase in magnitude of brain activity } \\
\text { from } \mathrm{T} 1 \text { to } \mathrm{T} 2 \text { only in } \mathrm{BC} \text { with } \mathrm{CIA} \text {. Changes } \\
\text { in brain activity correlated with changes } \\
\text { in processing speed. Pattern of change in } \\
\text { brain activity before and after CTx varies } \\
\text { according to pre-treatment menopausal } \\
\text { status }\end{array}$ \\
\hline $\begin{array}{l}\text { Dumas et al. } \\
2013[53]\end{array}$ & $\begin{array}{l}9 \text { BCS CTX+A: } \\
57.10 \pm 8.6\end{array}$ & $\begin{array}{l}\text { C: } 100 \% ; \text { T: } 89 \% \\
\text { A: } 44 \%\end{array}$ & $\begin{array}{l}\text { L: T1: before } C T x+; \\
\text { T2: } 1 \text { month after; } \\
\text { T3: } 1 \text { year after } \\
\text { CTX+ }\end{array}$ & fMRI; n-back task & $\begin{array}{l}\text { Decreased functional connectivity } 1 \text { month } \\
\text { after CTx+, partially returned to baseline } \\
\text { in the dorsal attention network } 1 \text { year } \\
\text { after CTx+. Decreased connectivity in the } \\
\text { default mode network at T1 an T2. Increase } \\
\text { in subjective memory complaints one } \\
\text { month and } 1 \text { year after CTx }\end{array}$ \\
\hline $\begin{array}{l}\text { Askren et al. } \\
2014[54]\end{array}$ & $\begin{array}{l}28 \text { BSC CTX+A: } 50 \\
\pm 10 ; 37 \text { BCS CTX- } \\
\text { A: } 53 \pm 9 ; 32 \text { HC A: } \\
50 \pm 9\end{array}$ & $\begin{array}{l}\text { AC-P: } 79 \% ; C-D: \\
18 \% ; A C: 3 \%\end{array}$ & $\begin{array}{l}\text { L: } T 1 \text { : before } C T x \text {; } \\
\text { T2: } 1-5 \text { months } \\
\text { after CTX }\end{array}$ & $\begin{array}{l}\mathrm{fMRI} \text {; verbal } \\
\text { working memory } \\
\text { task }\end{array}$ & $\begin{array}{l}\text { Greater pre-treatment fatigue in CTx+ than } \\
\text { in } \mathrm{HC} \text { and compromised neural response } \\
\text { characterized by higher spatial variance in } \\
\text { executive network activity in CTx+ than in } \\
\mathrm{CTX} \text {. Pre-treatment neural inefficiency in } \\
\text { executive network was a better predictor } \\
\text { of postchemotherapy cognitive and fatigue } \\
\text { complaintes than chemotherapy per se }\end{array}$ \\
\hline $\begin{array}{l}\text { Deprez et al. } \\
2014 \text { [55] }\end{array}$ & $\begin{array}{l}18 \text { BC CTX+ A: } 43.7 \\
\pm 4.3 ; 16 \text { BC CTX- A: } \\
44.3 \pm 4.7 ; 17 \text { HC A: } \\
40.7 \pm 6.0\end{array}$ & $\begin{array}{l}\text { FEC-T: } 94 \% ; \text { FEC: } \\
6 \%\end{array}$ & $\begin{array}{l}\text { L; T1: before CTX+; } \\
\text { T2: 4-6 months } \\
\text { after CTx; }\end{array}$ & $\begin{array}{l}\text { fMRI; multitask } \\
\text { paradigm }\end{array}$ & $\begin{array}{l}\text { Decreased activation in the multitasking } \\
\text { network in } \mathrm{T} 2 \text { compared to } \mathrm{T} 1 \text { in } \mathrm{BCS} C \mathrm{CT}+\text {. } \\
\text { No differences between groups at T1. In } \\
\text { BCS CTX+ increase of cognitive complaints } \\
\text { in } \mathrm{T} 2\end{array}$ \\
\hline $\begin{array}{l}\text { Nudelman } \\
\text { et al. } 2014 \\
{[56]}\end{array}$ & $\begin{array}{l}27 \mathrm{BC} C T \mathrm{x}+\mathrm{A}: 49.9 \\
\pm 7.6 ; 26 \mathrm{BC} C \mathrm{CT}-\mathrm{A}: \\
52.0 \pm 8.9 ; 26 \mathrm{HC} \text { A: } \\
48.4 \pm 10.1\end{array}$ & $\begin{array}{l}\text { AC-P: } 30 \% \\
\text { AC: } 30 \% \\
\text { A+carboplatine: } \\
22 \%\end{array}$ & $\begin{array}{l}\mathrm{L} ; \mathrm{T} 1 \text { : after surgery } \\
\text { before other } \\
\text { treatments; } \mathrm{T2} \text { : } \\
1 \text { month after CTx } \\
\text { or yoked intervals }\end{array}$ & $\begin{array}{l}\text { pulsed arterial } \\
\text { spin labeling } \mathrm{MRI} \text {; } \\
\text { VBM }\end{array}$ & $\begin{array}{l}\text { No differences in baseline perfusion } \\
\text { between groups. Increased perfusion } \\
1 \text { month after CTx compared to baseline } \\
\text { in right precentral gyrus }\end{array}$ \\
\hline
\end{tabular}


Table 4. Cont.

\begin{tabular}{|c|c|c|c|c|c|}
\hline Study & Population & $\begin{array}{l}\text { Major CTx } \\
\text { treatment }\end{array}$ & Design & NI/NP methods & Major findings \\
\hline $\begin{array}{l}\text { Kesler et al. } \\
2015 \text { [16] }\end{array}$ & $\begin{array}{l}20 \text { BCS ACTX+ A: } 52 \\
\pm 7.6 ; 19 \text { BCS CTx+ } \\
\text { A: } 53 \pm 8.7 ; 23 \text { BC } \\
\text { CTx- A: } 58 \pm 7.9\end{array}$ & $\begin{array}{l}\text { ACTX+: AC-P: } 74 \% \\
\text { AC: } 10 \% ; \text { CAF } 5 \% \\
\text { CTX-: CP: } 79 \% \\
\text { CMF: } 11 \%\end{array}$ & $\begin{array}{l}\text { CS; TSCTx: } \\
\text { CTX+AC: } 2.2 \pm 1.5 \\
\text { years; TSCTx: } \\
\text { CTx+: } 2.1 \pm 1.6 \\
\text { years }\end{array}$ & $\begin{array}{l}\text { fMRI; The Hopkins } \\
\text { Verbal Learning } \\
\text { Test-Revised }\end{array}$ & $\begin{array}{l}\text { Lower verbal memory performance } \\
\text { (immediate and delayed recall), lower left } \\
\text { precuneus connectivity in } \mathrm{BC} \text { treated with } \\
\text { anthracycline-based CTx compared to BC } \\
\text { treated with non-anthracycline regiments } \\
\text { and } \mathrm{HC}\end{array}$ \\
\hline $\begin{array}{l}\text { Menning } \\
\text { et al. } 2015 \\
{[58]}\end{array}$ & $\begin{array}{l}32 \mathrm{BC} C T \mathrm{X}+\mathrm{A}: 50.2 \\
\pm 9.2 ; 33 \mathrm{BC} C T \mathrm{x}-\mathrm{A}: \\
52.4 \pm 7.3 ; 38 \mathrm{HC} \mathrm{A}: \\
50.1 \pm 8.7\end{array}$ & antracycline & CS; before CTx & $\begin{array}{l}\text { fMRI; Tower of } \\
\text { London; Paired } \\
\text { Associates } \\
\text { paradigm }\end{array}$ & $\begin{array}{l}\text { Hyperactivation in prefrontal area with } \\
\text { increasing task difficulty on a planning } \\
\text { task in both } \mathrm{BC} \text { groups compared to } \mathrm{HC} \text {, } \\
\text { but not during a memory task. Observed } \\
\text { changes were associated with symptoms } \\
\text { of fatigue }\end{array}$ \\
\hline $\begin{array}{l}\text { Piccirillo } \\
\text { et al. } 2015 \\
{[17]}\end{array}$ & $\begin{array}{l}15 \text { BCS CTx+ with } \\
\text { SRCI A: 54; } 13 \text { BC } \\
\text { CTx+ without SRCI } \\
\text { A: } 52\end{array}$ & $\begin{array}{l}\text { anthracyline } \\
\text { and/or tacane }\end{array}$ & $\begin{array}{l}\mathrm{CS} ; \mathrm{TSCTx}>30 \\
\text { days }\end{array}$ & rs-fcMRI & $\begin{array}{l}\text { Disrupted resting state functional } \\
\text { connectivity only in BCS women who self- } \\
\text { reported cognitive impairment }\end{array}$ \\
\hline $\begin{array}{l}\text { Stouten- } \\
\text { Kemperman } \\
\text { et al. } 2015 \\
{[57]}\end{array}$ & $\begin{array}{l}17 \text { BCS HCTX+A: } \\
56.3 \pm 5.5 ; 15 \text { BCS } \\
\text { CTX+ A: } 59.8 \pm 6.3 \\
15 \text { BCS RT A: } 58.2 \\
\pm 5.8 ; 27 \text { HC A: } \\
60.31 \pm 4.8\end{array}$ & $\begin{array}{l}\text { FEC: } 100 \% \text { or FEC- } \\
\text { CTC: } 100 \%\end{array}$ & $\begin{array}{l}\text { CS; TSCTx: } 11,5 \\
\text { years post CTx }\end{array}$ & $\begin{array}{l}\text { fMRI; Tower of } \\
\text { London; Paired } \\
\text { Associates } \\
\text { paradigm }\end{array}$ & $\begin{array}{l}\text { Hypoactivation in task-related prefrontal } \\
\text { and parietal areas in both CTx+ groups } \\
\text { compared to RT group. In HCTx+ } \\
\text { hypoactivation more pronounced as well } \\
\text { as worse task performance than in CTx+ }\end{array}$ \\
\hline $\begin{array}{l}\text { Jung et al. } \\
2016 \text { [18] }\end{array}$ & $\begin{array}{l}28 \text { BCS CTX }+ \text { A: } \\
49.68 \pm 9.74 ; 34 \\
\text { BCS CTX-A: } 53.94 \\
\pm 8.42 ; 30 \text { HC A: } \\
51.13 \pm 8.47\end{array}$ & $\begin{array}{l}\text { AC-T: } 79 \% ; \text { DC: } \\
18 \% ; A C: 4 \%\end{array}$ & $\begin{array}{l}\text { L: T1: before } \mathrm{CTX}+\text {; } \\
\text { T2: } 1 \text { month after } \\
\text { CTx; T3: } 7 \text { months } \\
\text { after CTx }\end{array}$ & $\begin{array}{l}\text { fMRI; Verbal } \\
\text { Working Memory } \\
\text { Task }\end{array}$ & $\begin{array}{l}\text { Changes in frontoparietal executive } \\
\text { network, cognitive complaints at } \\
\text { T3. Higher spatial variance (neural } \\
\text { inefficiency) in executive network in CTx+ } \\
\text { than in CT- and HC }\end{array}$ \\
\hline \multicolumn{6}{|c|}{ 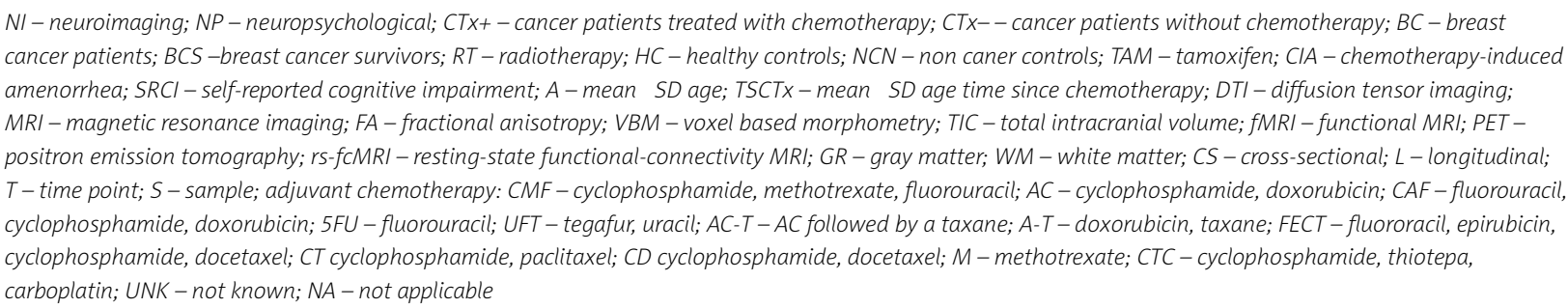 } \\
\hline
\end{tabular}

memory using the $n$-back paradigm. It was shown that the more the task was taxing to the working memory, the greater was the scope of activation of brain areas (bilateral stimulation of frontal and parietal areas) in the sister treated with CTx compared to the healthy one. However, no significant differences in the task performance level were observed [64].

The obtained results indicate that in order to enable the adequate performance level of a task by the twin treated with CTx, it was necessary to activate a greater area of neural networks, which most likely requires greater mental effort, reflected in the greater number of complaints about cognitive functioning $[64,68]$. It may be supposed that, if the task were made increasingly more taxing, at a certain level of difficulty the compensation for the deficits would be insufficient and the test results would become poorer [68]. The activation of larger areas of the brain in order to maintain the appropriate performance level in cognitive tasks was also confirmed by numerous studies on people aging normally [69-71].
The activation of compensatory mechanisms was also confirmed in a more recent longitudinal study carried out by McDonald, Conroy, Ahles, West, and Saykin [8], which assessed working memory using the $n$-back paradigm and brain activation using $\mathrm{fMRI}$ in women with breast cancer and in healthy ones. The measurements were taken three times: before chemotherapy, and one month, and one year after treatment. The performance level of n-back tasks did not differ significantly between groups; however, changes in activation patterns were observed in all three measurements, both during greater and lesser working memory-loaded tasks. Moreover, greater activation of prefrontal areas was found in the examinations before and one year after the treatment.

Thanks to compensatory neuroplasticity, the cognitive functioning of people treated with chemotherapy can be maintained on an unchanged or only slightly deteriorated level compared to their premorbid abilities. The studies on the levels of brain activation carried out with FMRI revealed that additional brain areas become involved in the performance of lower difficulty tasks, allowing their performance to remain within the norm. A deterioration in 
functioning becomes visible when the increasing difficulty exceeds the efficiency of compensatory mechanisms [68].

\section{Conclusions}

Based on the studies carried out using neuroimaging methods, it is possible to describe the cognitive deficits caused by adjuvant chemotherapy [72]. Specific, albeit small, structural changes and functional changes within the central nervous system are associated with the minor specific impairments of cognitive functions described in literature [72].

The changes in the activity of various cerebral regions in patients treated with chemotherapy indicate that the brain functions in an altered way, by activating new areas or creating new neural connections to reach the same cognitive efficiency. A greater expenditure of energy on mental activities can lead to increased fatigue and be associated with the deterioration in cognitive effectiveness and quality of life suffered by the patients [63]. Even though neuroimaging methods are not free from limitations, using them in $\mathrm{CRCl}$ studies in combination with self-descriptive and neuropsychological methods may yield a broader image of the described phenomenon [72].

\section{The authors declare no conflict of interest.}

\section{References}

1. Wefel JS, Vardy J, Ahles T, Schagen SB. International Cognition and Cancer Task Force recommendations to harmonise studies of cognitive function in patients with cancer. Lancet Oncol 2011; 12: 703-8.

2. Lindner OC, Phillips B, McCabe MG, Mayes A, Wearden A, Varese F, Talmi D. A meta-analysis of cognitive impairment following adult cancer chemotherapy. Neuropsychology 2014; 28: 726-40.

3. Bruno J, Hosseini SM, Kesler S. Altered resting state functional brain network topology in chemotherapy-treated breast cancer survivors. Neurobiol Dis 2012; 48: 329-38.

4. Conroy SK, McDonald BC, Smith DJ, et al. Alterations in brain structure and function in breast cancer survivors: effect of post-chemotherapy interval and relation to oxidative DNA damage. Breast Cancer Res Treat 2013; 137: 493-502.

5. de Ruiter MB, Reneman L, Boogerd W, Veltman DJ, van Dam FS, Nederveen AJ, Boven E, Schagen SB. Cerebral hyporesponsiveness and cognitive impairment 10 years after chemotherapy for breast cancer. Hum Brain Mapp 2011; 32: 1206-9.

6. Inagaki M, Yoshikawa E, Matsuoka Y, et al. Smaller regional volumes of brain gray and white matter demonstrated in breast cancer survivors exposed to adjuvant chemotherapy. Cancer 2007; 109: 146-56.

7. Kesler SR, Kent JS, O'Hara R. Prefrontal cortex and executive function impairments in primary breast cancer. Arch Neurol 2011; 68: 1447-53.

8. McDonald BC, Conroy SK, Ahles TA, West JD, Saykin AJ. Alterations in brain activation during working memory processing associated with breast cancer and treatment: a prospective functional magnetic resonance imaging study. J Clin Oncol 2012; 30: 2500-8.

9. Silverman DH, Dy CJ, Castellon S, et al. Altered frontocortical, cerebellar, and basal ganglia activity in adjuvant-treated breast cancer survivors 5-10 years after chemotherapy. Breast Cancer Res Treat 2007; 103: 303-11.

10. Bergouignan L, Lefranc JP, Chupin M, Morel N, Spano JP, Fossati P. Breast cancer affects both the hippocampus volume and the episodic autobiographical memory retrieval. PLoS One 2011; 6: e25349.

11. Deprez S, Amant F, Smeets A, et al. Longitudinal assessment of chemotherapy-induced structural changes in cerebral white matter and its correlation with impaired cognitive functioning. J Clin Oncol 2012; 30: 274-81.
12. McDonald BC, Conroy SK, Smith DJ, West JD, Saykin AJ. Frontal gray matter reduction after breast cancer chemotherapy and association with executive symptoms: A replication and extension study. Brain Behav Immun 2013; 30 Suppl: S117-25.

13. Kesler SR, Janelsins M, Koovakkattu D, Palesh O, Mustian K, Morrow G, Dhabhar FS. Reduced hippocampal volume and verbal memory performance associated with interleukin- 6 and tumor necrosis factor-alpha levels. Brain Behav Immun 2013; 30 Suppl: S109-16.

14. Kesler SR, Bennett FC, Mahaffey ML, Spiegel D. Regional brain activation during verbal declarative memory in metastatic breast cancer. Clin Cancer Res 2009; 15: 6665-73.

15. López Zunini RA, Scherling C, Wallis N, Collins B, Mackenzie J, Bielajew C, Smith AM. Differences in verbal memory retrieval in breast cancer chemotherapy patients compared to healthy controls: a prospective fMRI study. Brain Imaging Behav 2013; 7: 460-77.

16. Kesler SR, Blayney DW. Neurotoxic effects of anthracycline- vs nonanthracycline-based chemotherapy on cognition in breast cancer survivors. JAMA Oncol 2016; 2: 185-92.

17. Piccirillo JF, Hardin FM, Nicklaus J, et al. Cognitive impairment after chemotherapy related to atypical network architecture for executive control. Oncology 2015; 88: 360-8.

18. Jung MS, Zhang M, Askren MK, et al. Cognitive dysfunction and symptom burden in women treated for breast cancer: a prospective behavioral and fMRI analysis. Brain Imaging Behav 2016; doi: 10.1007/ s11682-016-9507-8.

19. Staat K, Segatore M. The phenomenon of chemo brain. Clin J Oncol Nursing 2005; 9: 713-21.

20. Vardy J, Tannock I. Cognitive function after chemotherapy in adults with solid tumours. Crit Rev Oncol Hematol 2007; 63: 183-202.

21. Taillibert S. Is systemic anti-cancer therapy neurotoxic? Does chemo brain exist? And should we rename it? Adv Exp Med Biol 2010; 678: 86-95.

22. Ahles TA, Saykin AJ. Candidate mechanisms for chemotherapy-induced cognitive changes. Nat. Rev. Cancer 2007; 7: 192-201.

23. Dietrich J, Han R, Yang Y, Mayer-Pröschel M, Noble M. CNS progenitor cells and oligodendrocytes are targets of chemotherapeutic agents in vitro and in vivo. J Biol 2006; 5: 22.

24. Tuxen MK, Hansen SW. Neurotoxicity secondary to antineoplastic drugs. Cancer Treat Rev 1994; 20: 191-214.

25. Troy L, McFarland K, Littman-Power S, Kelly BJ, Walpole ET, Wyld D, Thomson D. Cisplatin-based therapy: a neurological and neuropsychological review. Psychooncology 2000; 9: 29-39.

26. Ahles TA, Saykin AJ, Noll WW, Furstenberg CT, Guerin S, Cole B, Mott LA. The relationship of APOE genotype to neuropsychological performance in long-term cancer survivors treated with standard dose chemotherapy. Psychooncology 2003; 12: 612-9.

27. Seigers R, Timmermans J, van der Horn HJ et al. Methotrexate reduces hippocampal blood vessel density and activates microglia in rats but does not elevate central cytokine release. Behav Brain Res 2010; 207: 265-72.

28. Barton D, Loprinzi C. Novel approaches to preventing chemotherapy-induced cognitive dysfunction in breast cancer: the art of the possible. Clin Breast Cancer 2002; 3 Suppl 3: S121-7.

29. Fillit HM, Butler RN, O'Connell AW, et al. Achieving and maintaining cognitive vitality with aging. Mayo Clin Proc 2002; 77: 681-96.

30. Ahles TA. Do systemic cancer treatments affect cognitive function? Lancet Oncol 2004; 5: 270-1.

31. Koppelmans V, Breteler MM, Boogerd W, Seynaeve C, Schagen SB.. Late effects of adjuvant chemotherapy for adult onset non-CNS cancer; cognitive impairment, brain structure and risk of dementia. Crit Rev Oncol Hematol 2013; 88: 87-101.

32. Small BJ, Rawson KS, Walsh E, Jim HS, Hughes TF, Iser L Andrykowski MA, Jacobsen PB. Catechol-O-methyltransferase genotype modulates cancer treatment-related cognitive deficits in breast cancer survivors. Cancer 2011; 117: 1369-76.

33. Vardy J, Wefel JS, Ahles T, Tannock IF, Schagen SB. Cancer and cancer-therapy related cognitive dysfunction: an international perspective from the Venice cognitive workshop. Ann Oncol 2007; 19: 623-9.

34. Yoshikawa E, Matsuoka Y, Inagaki M et al. No adverse effects of adjuvant chemotherapy on hippocampal volume in Japanese breast cancer survivors. Breast Cancer Res Treat 2005; 92: 81-4.

35. Abraham J, Haut MW, Moran MT, Filburn S, Lemiuex S, Kuwabara H. Adjuvant chemotherapy for breast cancer: effects on cerebral white 
matter seen in diffusion tensor imaging. Clin Breast Cancer 2008; 8: 88-91.

36. Deprez S, Amant F, Yigit R, et al. Chemotherapy-induced structural changes in cerebral white matter and its correlation with impaired cognitive functioning in breast cancer patients. Hum Brain Mapp 2011; 32: 480-93.

37. McDonald BC, Conroy SK, Ahles TA, West JD, Saykin AJ. Gray matter reduction associated with systemic chemotherapy for breast cancer: a prospective MRI study. Breast Cancer Res Treat 2010; 123: 819-28.

38. de Ruiter MB, Reneman L, Boogerd W, et al. Late effects of high-dose adjuvant chemotherapy on white and gray matter in breast cancer survivors: Converging results from multimodal magnetic resonance imaging. Hum. Brain Mapp 2012; 33: 2971-83.

39. Koppelmans V, De Ruiter MB, Van Der Lijn F, et al. Global and focal brain volume in long-term breast cancer survivors exposed to adjuvant chemotherapy. Breast Cancer Res Treat 2012; 132: 1099-106.

40. Hosseini SM, Koovakkattu D, Kesler SR. Altered small-world properties of gray matter networks in breast cancer. BMC Neurol 2012; 12: 28.

41. Scherling C, Collins B, MacKenzie J, Lepage C, Bielajev C, Smith A. Structural brain differences in breast cancer patients compared to matched controls prior to chemotherapy. Int J Biol 2012; 4: 3.

42. Lepage C, Smith AM, Moreau J, Barlow-Krelina E, Wallis N, Collins B, MacKenzie J, Scherling C. A prospective study of grey matter and cognitive function alterations in chemotherapy-treated breast cancer patients. Springerplus 2014; 3: 444

43. Koppelmans V, Vernooij MW, Boogerd W, Seynaeve C, Ikram MA, Breteler MM, Schagen SB. Prevalence of cerebral small-vessel disease in long-term breast cancer survivors exposed to both adjuvant radiotherapy and chemotherapy. J Clin Oncol 2015; 33: 588-93.

44. Kreukels BP, Schagen SB, Ridderinkhof KR, Boogerd W, Hamburger HL, van Dam FS. Electrophysiological correlates of information process ing in breast-cancer patients treated with adjuvant chemotherapy. Breast Cancer Res Treat 2005; 94: 53-61.

45. Kreukels BP, Schagen SB, Ridderinkhof KR, Boogerd W, Hamburger HL, Muller MJ, van Dam FS. Effects of high-dose and conventional-dose adjuvant chemotherapy on long-term cognitive sequelae in patients with breast cancer: an electrophysiologic study. Clin Breast Cancer 2006; 7: 67-78.

46. Ferguson RJ, McDonald BC, Saykin AJ, Ahles TA. Brain structure and function differences in monozygotic twins: possible effects of breast cancer chemotherapy. J Clin Oncol 2007; 25: 3866-70.

47. Kreukels BP, van Dam FS, Ridderinkhof KR, Boogerd W, Schagen SB. Persistent neurocognitive problems after adjuvant chemotherapy for breast cancer. Clin Breast Cancer 2008; 8: 80-7.

48. Cimprich B, Reuter-Lorenz P, Nelson J, et al. Prechemotherapy alterations in brain function in women with breast cancer. J Clin Exp Neuropsychol 2010; 32: 324-31.

49. Scherling C, Collins B, Mackenzie J, et al. Pre-chemotherapy differences in visuospatial working memory in breast cancer patients compared to controls: an FMRI study. Front Hum Neurosci 2011; 5: 122.

50. Scherling C, Collins B, Mackenzie J, Bielajev C, Smith A. Prechemotherapy differences in response inhibition in breast cancer patients compared to controls: a functional magnetic resonance imaging study. J Clin Exp Neuropsychol 2012; 34: 543-60.

51. Kesler SR, Wefel JS, Hosseini SM, Cheung M, Watson CL, Hoeft F. Default mode network connectivity distinguishes chemotherapy-treat ed breast cancer survivors from controls. Proc Natl Acad Sci U S A 2013; 110: 11600-5.

52. Conroy SK, McDonald BC, Ahles TA, West JD, Saykin AJ. Chemotherapy-induced amenorrhea: a prospective study of brain activation changes and neurocognitive correlates. Brain Imaging Behav 2013; 7: 491-500.

53. Dumas JA, Makarewicz J, Schaubhut GJ, Devins R, Albert K, Dittus K, Newhouse PA. Chemotherapy altered brain functional connectivity in women with breast cancer: a pilot study. Brain Imaging Behav 2013; 7: 524-32.

54. Askren MK, Jung M, Berman MG, et al. Neuromarkers of fatigue and cognitive complaints following chemotherapy for breast cancer: a prospective fMRI investigation. Breast Cancer Res Treat 2014; 147: $445-55$

55. Deprez S, Vandenbulcke M, Peeters R, Emsell L, Smeets A, Christiaens MR, Amant F, Sunaert S. Longitudinal assessment of chemothera- py-induced alterations in brain activation during multitasking and its relation with cognitive complaints. J Clin Oncol 2014; 32: 2031-8.

56. Nudelman KN, Wang Y, McDonald BC, et al. Altered cerebral blood flow one month after systemic chemotherapy for breast cancer: a prospective study using pulsed arterial spin labeling MRI perfusion. PLoS One 2014; 9: e96713.

57. Stouten-Kemperman MM, de Ruiter MB, Boogerd W, Veltman DJ, Reneman L, Schagen SB. Very late treatment-related alterations in brain function of breast cancer survivors. J Int Neuropsychol Soc 2015; 21: 50-61.

58. Menning S, de Ruiter MB, Veltman DJ, Koppelmans V, Kirschbaum C, Boogerd W, Reneman L, Schagen SB. Multimodal MRI and cognitive function in patients with breast cancer prior to adjuvant treatment the role of fatigue. Neuroimage Clin 2015; 7: 547-54.

59. Ashburner J, Friston KJ. Voxel-based morphometry - the methods. Neuroimage 2000; 11 (6 Pt 1): 805-21.

60. Le Bihan D, Mangin JF, Poupon C, Clark CA, Pappata S, Molko N, Chabriat $\mathrm{H}$. Diffusion tensor imaging: concepts and applications. J Magn Reson Imaging 2001; 13: 534-46.

61. McDonald BC, Saykin AJ. Alterations in brain structure related to breast cancer and its treatment: chemotherapy and other considerations. Brain Imaging Behav 2013; 7: 374-87.

62. Lepage M, Habib R, Tulving E. Hippocampal PET activations of memory encoding and retrieval: the HIPER model. Hippocampus 1998; 8: $313-22$.

63. Scherling CS, Smith A. Opening up the window into and "chemobrain": A neuroimaging review. Sensors (Switzerland) 2013; 13: 3169203.

64. Ferguson CJ. The good, the bad and the ugly: a meta-analytic review of positive and negative effects of violent video games. Psychiatr Q 2007; 78: 309-16.

65. Kreukels BP, Hamburger HL, de Ruiter MB, van Dam FS, Ridderinkhof KR, Boogerd W, Schagen SB. ERP amplitude and latency in breast cancer survivors treated with adjuvant chemotherapy. Clin Neurophysiol 2008; 119: 533-41.

66. Honea RA, Vidoni E, Harsha A, Burns JM. Impact of APOE on the Healthy Aging Brain: A Voxel-Based MRI and DTI Study. J Alzheimers Dis 2008; 18: 553-64.

67. Persson J, Lind J, Larsson A, et al. Altered brain white matter integrity in healthy carriers of the APOE epsilon4 allele: a risk for AD? Neurology 2006; 66: 1029-33.

68. Reuter-Lorenz PA, Cimprich B. Cognitive function and breast cancer: Promise and potential insights from functional brain imaging. Breast Cancer Res Treat 2013; 137: 33-43.

69. Cappell KA, Gmeindl L, Reuter-Lorenz PA. Age differences in prefontal recruitment during verbal working memory maintenance depend on memory load. Cortex 2010; 46: 462-73.

70. Eyler LT, Sherzai A, Kaup AR, Jeste DV. A review of functional brain imaging correlates of successful cognitive aging. Biol Psychiatry 2011; 70: $115-22$.

71. Schneider-Garces NJ, Gordon BA, Brumback-Peltz CR, et al. Span, $\mathrm{CRUNCH}$, and beyond: working memory capacity and the aging brain. J Cogn Neurosci 2010; 22: 655-69.

72. Raffa RB. Imaging as a means of studying chemotherapy-related cognitive impairment. Adv Exp Med Biol 2010; 678: 70-6.

\section{Address for correspondence}

\section{Paulina Andryszak}

Institute of Psychology

Kazimierz Wielki University in Bydgoszcz

Staffa 1

85-867 Bydgoszcz, Poland

e-mail: pandryszak@gmail.com

Submitted: 13.12 .2015

Accepted: $\quad 30.09 .2016$ 